\title{
Compassionate Conversations
}

Sharryn Gardner, Dominic Bray

Southport and Ormskirk NHS Trust

\begin{abstract}
Staff engagement is much more than just a bonus in any organisation. CQC data shows that it is very clearly linked to positive results in both patient and staff outcomes (fewer complaints, improved safety, reduced sickness, fewer accidents, and more as per Michael West). Staff engagement may seem nebulous but is in fact measured routinely annually in the National Staff Survey. The problem is that often Trust Boards with poor Staff Survey results may struggle to increase staff engagement as staff see management initiatives as 'management fads' or 'tick-box exercises' purely for targets, not their own benefit.
\end{abstract}

Compassionate Conversations are a ground-level initiative focused primarily on supporting and motivating individual staff as the primary focus. This allows the benefits to patients and in Human Resources to be an unspoken anticipated benefit. They are led by a Psychologist and Consultant in a coaching supportive atmosphere in an open or selected group.

The Conversations have been rated $9 / 10$ or higher by $64 \%$ of participants, while $75 \%$ of all participants voluntarily provided feedback. Feedback initially was that Conversations were too clinically-focused and further Conversations were more wide-ranging in topic and included departmental roadshows.

\section{Problem}

The problem is that some Organisations (Acute UK Hospital Trusts) may have very poor Staff Survey results and have no obvious way of being able to address this. The poor results may be mirrored in suboptimal patient and HR results, though no organisation is perfect and almost all could benefit from further positive staff engagement.

Pulse Check results act as a snapshot in time of staff views of their organisation. These can be initiated within programmes such as Listening into Action or Scope for Change which seek to empower staff to make positive changes in teams across the organisation. The assumption from West's work mapping CQC data and National Staff Survey data over years is that there will be a gap between the current performance and the potential performance. West has estimated this at around 40 lives saved per District General Hospital where a Trust Board is engaged with staff for example.

Staff may suffer as there is likely to be higher stress and sickness levels as well as patient complaints. Patients may suffer from less compassionate care, more safety lapses, and less efficient care. Management may suffer due to poorer results and an inability to demonstrate anticipated improvement with apparently appropriate staff training.

The situation is maintained as staff who are not engaged may be sceptical at initiatives to improve engagement as phoney.

\section{Background}

Schwartz Rounds in the States were developed to combat this potential gap in compassion by having regular whole-hospital meetings to discuss potentially emotive issues. Evidence shows that staff gain support from others and mutual respect between teams. This then translates into lower mortality and morbidity as well as better results in HR metrics such as staff sickness and accidents at work. Michael West has demonstrated this direct correlation reliably over 10 years from CQC and National Staff Survey data.

Compassion was the biggest theme in the recent Francis Report into the crisis at Mid Staffordshire, though it can seem quite nebulous as a metric which can be addressed. The Point of Care Foundation (previously the King's Fund) has taken on a role of rolling out this or a similar program as far as possible. They have not only introduced this model, they have reported extensively on the early and ongoing results.

Currently there are no other similar options and for many Trusts (despite any recognised need to promote compassion) Schwartz Rounds can be prohibitively expensive. They have a tried and tested formula, and to remain accredited have some constraints on how to run them.

\section{Baseline measurement}

There is no obvious direct measure of staff engagement, though this is a small part of the overall National Staff Survey - there is an overall section on staff engagement with 4 sub-questions.

Schwartz Rounds in the States were developed to combat this by having regular whole-hospital meetings to discuss these emotive issues. They pre-dated Francis by around 20 years and had published evidence on their benefits almost 10 years ago. Evidence shows that staff gain support from others and mutual respect 
between teams. Compassion was overwhelmingly the biggest theme in the recent Francis Report into the crisis at Mid Staffordshire. The Point of Care Foundation has taken on a role of rolling out this or a similar program as far as possible.

This then translates into lower mortality and morbidity. We know this as Michael West has spent 10 years mapping National Staff Survey data against CQC outcome and quality data. It seems that they are directly related implying that training and multiple management initiatives may not be enough without Organisational support and engagement for individuals and an appreciation of their efforts.

See supplementary file: ds3373.pdf - "Schwartz Rounds Summary"

\section{Design}

The intervention stemmed from the Director of Nursing hearing about Schwartz Rounds and wanting something similar.

The basic Compassionate Conversation was developed by a core Solution Focus Practice trained group who would act as facilitators. They had experience of using the various elements with success in other settings and felt that this combination should work well.

The intervention started very quickly within a Trust-adopted commercial process called Listening Into Action which had a timescale of 20 weeks though it was always planned to be a longterm plan and to become embedded.

The intervention is an ongoing programme of regular whole-hospital meetings and departmental roadshows. These are pitched directly at all groups of staff as individuals and for their own needs as much as for those of the patients. The best events have had the most diverse groups of staff including domestics, porters, the CEO, Trust Governors, HR staff and others. Equally small group events work well in small teams where staff bond and feel safe as there are no outsiders present.

Like Schwartz Rounds the monthly whole-hospital events last one hour. Coffee and doughnuts (always doughnuts as these have significance) are provided. The events are currently all led by a Psychologist and Consultant, both of whom use a relaxed and at times humorous approach. Participants are encouraged to feel free to say politically incorrect things and use gallows humour within the confines of the safe environment. Unlike Schwartz Rounds, we have chosen coffee lounge-like areas so that participants feel relaxed and are primed to mix and chat. As the event starts, participants are reminded that any patient or staff details in the room are to be treated confidentially. Equally, they can feel free to express emotions as there is strict a policy of 'what happens in Vegas, stays in Vegas' (supported by posters as participants enter the room). Unless there is a genuine serious and material risk, no information will be acted upon outside the meeting. There is an opening part describing why our own needs are important in order to provide good care and how healthy it is to focus on this. that pair with a member of staff they didn't already know. They are then asked to spend several minutes (in turn) asking each other what they feel proud of doing in the last couple of weeks - even if that is being able to actually get into work. Essentially 'what have you done lately to make you feel proud?'. Surprisingly, when participants have often been cajoled into coming, this is often a liberating experience and there is a palpable buzz in the room. They then ask each other how did they do that and did they have to learn how to, or work at it.

Following this participants are warmed up and have developed a 'yes-set' as it is called in selling. They are willing to stay engaged. The facilitators then lead a discussion loosely based on a predetermined theme such as where there is no medical fix, describing your most memorable colleague (or patient), the aftermath of a serious incident and others. Participants often jump right in and are very frank, honest, and candid. After about 20-30 minutes the meeting is brought to a close, everyone is reminded about confidentiality, and encouraged (not required) to leave feedback on prepared forms.

\section{Strategy}

The feedback was used in realtime as well as facilitator's perceptions during events to modify the basic template.

\section{PDSA cycle 1}

The first event was very poorly attended and we refocused on promoting direct benefits to staff.

The second event was much better attended following managers highlighting the need to attend and giving impetus to managers to allow staff time to attend. Promotional materials became very visual, very bold, and very individual-focused.

\section{PDSA cycle 2}

Staff highlighted other staff who couldn't attend and that themes were too clinically focused. Roadshows were held within departments and developed with staff to find out what they wanted out of them. The themes were made more universal and roadshows were tailored partly to showcasing teams as well as exploring difficulties. This was very successful and is being expanded exponentially.

\section{Results}

Unfortunately due to the tight timescale with Listening Into Action, the repeat Pulse Check data is not yet available and the next National Staff Survey is currently in process.

$10 \%$ of staff have attended either a roadshow or Compassionate Conversation.

$75 \%$ left some form of (voluntary) feedback. 


\section{BMJ Quality Improvement Reports}

The average (mean) rating on a $0-10$ scale was 8.9 and $64 \%$ scored the event 9 or 10 out of 10 .

\section{Lessons and limitations}

The process started through the Listening Into Action programme which provided an Executive Sponsor as well as a timeline to get a sponsor group and get started. There was no funding allocated specifically. In the initial stages the facilitators were able to shuffle other commitments to accommodate these roles and going forward as more and more are requested, this will need to be more formally recognised through job planning which will then have resource implications. The success of the project has led to group and external Compassionate Conversations and roadshows.

Each individual Compassionate Conversation cost around £20 for refreshments - staff time was not accounted for, though they were scheduled at lunchtime.

\section{Conclusion}

The results did clearly show that staff rated the sessions very highly. It is not yet clear if that translates into a sustained effect and how long it will take (if at all) to register in better CQC results and HR metrics. Some staff have attended more than one event and many who attend have heard about them from others.

Facilitators, staff, the Sponsor Group, and The Executive Board are universally positive and it is continuing with spread into different projects and settings.

The initial project was 20 weeks in line with the Listening Into Action timescale, though from the initiation this project was always planned to become embedded and routine, and the Board are very supportive of that. It continues under the locally driven Scope for Change umbrella and the diversity of the settings continues to lead to further experimentation and diversification. The underlying model (Solution-Focused Practice - a positive coaching-like model) is one which a core group in the Trust are gradually pushing into all areas of the hospital - patient contacts, appraisals, complaints, disciplinary procedures, and others. The long-term aim is to have the first Solution-Focused Integrated Care Organisation - one which succeeds and thrives in this difficult environment.

\section{References}

1. West M, Borrill CS, Dawson JF, Scully J, Carter M, Anelay $S$, et al. The link between the management of employees and patient mortality in acute hospitals. The International Journal of Human Resource Management.

2002;13(8):1299-1310.

2. Helen; Armstrong, Claire; West, Michael; Dawson, Jeremy. The impact of leadership and quality climate on hospital performance. International Journal for Quality in Health Care, Vol. 20, No. 6, 12.2008, p. 439-445.

3. Point of Care Foundation. Staff care: how to engage staff in the NHS and why it matters. Jan 2014.
www.pointofcarefoundation.org.uk/Downloads/Staff-

Report-2014.pdf.

4. Joanna Goodrich Supporting hospital staff to provide compassionate care: Do Schwartz Center Rounds work in English hospitals? J R Soc Med 2012 105: 117

5. Finlayson B. NHS morale. Singing the blues Serv J. 2002 Apr 4;112(5799):30-1.

\section{Declaration of interests}

Nothing to declare

\section{Acknowledgements}

Dominic Bray, Linda Lewis, Sharon Partington 